\title{
Optimization of the engineering surveys geodetic coordinate frame based on the Mercator projection use
}

\author{
Tran Thanh Son* \\ Hanoi University of Natural Resources and Environment Branch in Thanh Hoa Province, Vietnam
}

\begin{abstract}
The article proposes the use of a geodetic coordinate frame suitable for the reference plan geodetic networks of Vietnam used in engineering surveys. This framework minimizes distortion in horizontal spacing when going from ellipsoid to plane. In the course of the work, the parameters of the coordinate system and the height of the level surface were selected using the Universal Transverse Mercator (UTM). The equations used for the calculations are determined based on theoretical analysis and are easily applied in surveying.
\end{abstract}

\section{Introduction}

Geodetic reference networks are created for conducting geodetic design, construction and other types of engineering work. One of the main requirements when building a reference network is the observance of the principle "from the general to the particular" and the unity of the coordinate system. In Vietnam, the coordinates of geodetic networks points are defined in the national coordinate system VN-2000. The given coordinate system is based on the UTM transverse cylindrical Mercator projection on the WGS-84 ellipsoid starting at N00 at the Cadastre Institute in Hanoi. The distance of some territories in Vietnam from this axial meridian is more than $350 \mathrm{~km}$, therefore, significant deformations of the measured lengths are possible when switching to this coordinate system. In this regard, when creating a geodetic coordinate frame in construction, it is necessary to select the parameters of the coordinate system providing the least distortion of the lengths reduced to the plane.

\section{Matherials and Methods}

As a general principle, the measured values in the same geodetic network should be projected onto a selected coordinate plane (reference surface). The process of projecting a measurement on a plane includes two steps: projecting the measurement from the ground to

\footnotetext{
${ }^{*}$ Corresponding author: sonphuong85@mail.ru
} 
the surface of the reference ellipsoid and projecting from the reference ellipsoid onto the coordinate plane.

The length of a line measured on the Earth's surface is known to be taken into account with two corrections $[1-3]$ :

- ellipsoid altitude correction $\Delta S_{H}$ is calculated by the formula (Fig. 1):

$$
\Delta S_{H}=-\frac{H_{m}-H_{0}}{R_{m}} S^{\prime}
$$

where $S^{\prime}$ is the measured line length $A B ; H_{m}$ is the average measured line height; $H_{0}$ is the height of the reference ellipsoid above the general terrestrial ellipsoid; $R_{m}=6370 \mathrm{~km}$.

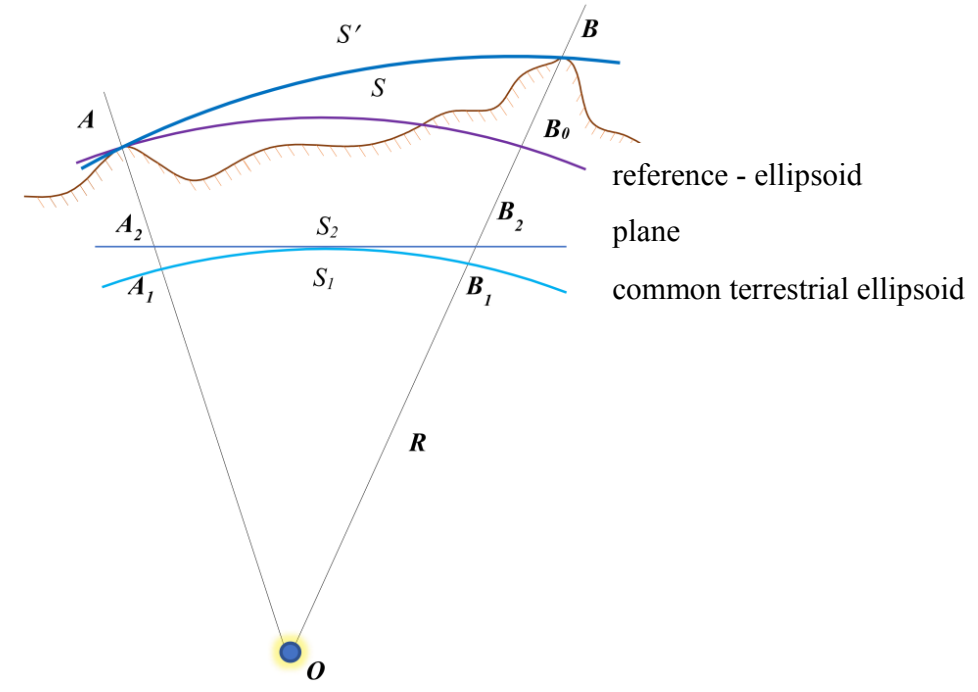

Fig. 1. The line projection onto the ellipsoid surface

- correction for transition to plane in UTM (or Gauss-Kruger) $\Delta S_{G}$ projection, calculated by the formula:

$$
\Delta S_{G}=\left(k_{0}-1+\frac{Y_{m}^{2}}{2 R_{m}^{2}}\right) S
$$

where: $S$ is the length of the ellipsoid surface line (the distance between the points on the reference ellipsoid); $Y_{m}=\left(\frac{Y_{a}+Y_{b}}{2}\right)$ is na average value of the starting and ending abscissas of the line point $A B$; $k_{0}$ is a scale factor on the axial meridian of the zone in the Gauss-Kruger projection $k_{0}=1$ and in the projection UTM $k_{0}=0.9996$ (six degree zone) or $k_{0}=0.9999$ (three-degree zone).

For a Gaussian projection, the line correction will have a positive indicator and an increase from the meridian axis to the edge of the projection zone. For the UTM projection, the correction will have a positive or negative indicator, depending on the relative position of the midpoint of the line compared to the meridian axis. 
To achieve the lowest distortion compared to the size of the network in the field, corrections to the line must be taken into account. Therefore, it is necessary to select the height of the projection surface $H_{0}$ and, accordingly, choose the meridian of the axis $L_{0}$. In documents for engineering and geodetic works [1-5], the instructions for choosing a coordinate system and a reference surface are often given as follows:

- selection of the actual height of the reference ellipsoid coincides with the average height of the construction area;

- meridians of the projection zone are selected depending on the projection and width of the projection zone. For Gaussian projection $(k=1)$ it is necessary to choose the meridian axis so that $Y_{m}=0$. In this case, the meridian axis of the projection of the median meridian of the construction site is selected. In UTM projection for the projection area $6^{\circ}(k=$ $0,9996)$ and zones $3^{\circ}(k=0,9999)$ are selected accordingly $Y_{m}= \pm 180 \mathrm{~km}$ and $Y_{m}= \pm 90$ $\mathrm{km}$.

When choosing a coordinate system as shown above, the correction $\Delta S_{G}$ will be of little importance when the midpoint of the line is near the projection zone meridian axis. When the midpoint of the line is far from the axial meridian, the correction $\Delta S_{G}$ can be significant. The influence of the meridian axis choice of the zone on the correction $\Delta S_{G}$ in Gaussian and UTM projections is shown below.

It follows from the formula (3):

$$
\frac{\Delta S_{m p}}{S}=k-1+\frac{Y_{m}^{2}}{2 \mathrm{R}_{\mathrm{m}}^{2}}
$$

Based on the formula (3), the relative values of the correction were determined for different mean values of the initial and final abscissas of the points, the results are shown in Table 1.

Table 1. Dependence between line length corrections and the average value of the starting and ending points abscissas

\begin{tabular}{|c|c|c|c|c|c|c|}
\hline \multirow{2}{*}{ Projection } & \multicolumn{5}{|c|}{ Relationship between line length corrections to plane $\frac{\Delta S_{m p}}{S}$} \\
\cline { 2 - 7 } & $\mathrm{Y}_{\mathrm{m}-20 \mathrm{~km}}$ & $\mathrm{Y}_{\mathrm{m}}-10 \mathrm{~km}$ & $\mathrm{Y}_{\mathrm{m}}-5 \mathrm{~km}$ & $\mathrm{Y}_{\mathrm{m}}+5 \mathrm{~km}$ & $\mathrm{Y}_{\mathrm{m}}+10 \mathrm{~km}$ & $\mathrm{Y}_{\mathrm{m}}+20 \mathrm{~km}$ \\
\hline $\begin{array}{c}\text { Gaussian } \\
\text { projection }\end{array}$ & $\frac{1}{203000}$ & $\frac{1}{812000}$ & $\frac{1}{3246000}$ & $\frac{1}{3246000}$ & $\frac{1}{812000}$ & $\frac{1}{203000}$ \\
\hline $\begin{array}{c}\text { UTM } \\
\text { Projection } \\
-3^{\circ}\end{array}$ & $-\frac{1}{25200}$ & $-\frac{1}{47300}$ & $-\frac{1}{91100}$ & $\frac{1}{89200}$ & $\frac{1}{43100}$ & $\frac{1}{20400}$ \\
\hline $\begin{array}{c}\text { UTM } \\
\text { Projection } \\
-6^{\circ}\end{array}$ & $-\frac{1}{11800}$ & $-\frac{1}{22800}$ & $-\frac{1}{44200}$ & $\frac{1}{46000}$ & $\frac{1}{22300}$ & $\frac{1}{10800}$ \\
\hline
\end{tabular}

The following conclusions can be drawn from Table 1:

- the correction of the line length to the plane in the Gaussian projection has a small and variable magnitude when the average value of the abscissa of the point increases in the direction from the meridian axis; 
- the corrections to the plane in the UTM projection are relatively large and change rapidly when the value of the line abscissa is deviated from the selected axial meridian. In addition, these corrections have the opposite indicator (minus) with a line on the right and left sides of the axial meridian. This leads to significant deformation of the geodetic network during construction.

To minimize line-to-plane correction $\Delta S_{m p}$ in the UTM projection, we offer our own approach to the choice of a coordinate system and a reference ellipsoid for a geodetic grid.

The proposed approach

The proposed approach consideres the selection of the axial meridian of the zone (middle meridian) for the engineering and geodetic works area and to add a correction for the deformation (distortion) of the axial meridian line length by 0 to the formula (2). The correction should be expressed in the form $\frac{T}{R_{m}} S$, then the length correction of the line to the plane will be calculated by the formula:

$$
\Delta S_{m p}=S\left(k-1+\frac{Y_{m}^{2}}{2 \mathrm{R}_{m}^{2}}+\frac{T}{R_{m}}\right),
$$

with the condition $\Delta S_{m p}=0$ by $\mathrm{Y}_{m}=0$,

$$
T=R_{m}(k-1) \text { can be defined. }
$$

In the UTM projection for the projection area $3^{\circ} \mathrm{T}=637 \mathrm{~m}$ is calculated, for the projection area $6^{\circ} \mathrm{T}=2548 \mathrm{~m}$ is calculated. Thus, in the proposed coordinate system, the selected axial meridian of the zone coincides with the average construction site. At the same time, it is necessary to select the height of the measured line (height of the reference ellipsoid) equal to $\left(H_{m}-H_{0}+T\right)$.

Thus, for the proposed coordinate system, the line length correction is calculated by the following formulas:

- ellipsoid altitude correction $\Delta \mathrm{SH}$, calculated by the expression

$$
\Delta S_{H}=-S \frac{T+H_{m}-H_{0}}{\mathrm{R}_{\mathrm{m}}} ;
$$

- correction for the transition to the plane is calculated by the formula (4).

In formulas (4) and (6) the coefficients $T$ and $k$ have the following meanings:

- with Gaussian projection, $T=0, k=1$;

- with UTM projection, projection area $3^{\circ}, k=0,9999, T=637 \mathrm{~m}$ and projection area $6^{\circ}, k=0,9996, T=2548 \mathrm{~m}$.

\section{Experimental calculation}

For verification, in the experimental section, the correction of the line length in the geodetic polygonometric network has been calculated. Assume that the network consists of 10 lines, the length of the line is $2 \mathrm{~km}$, the height of the points is $0 \mathrm{~m}$, the UTM coordinate system, the zone $3^{\circ}$ (see Fig. 2). 


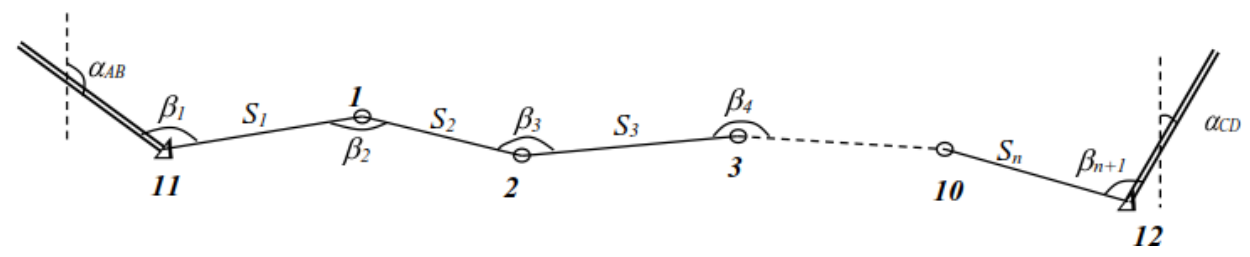

Fig. 2. Polygonometric grid for the experiment

Line length correction is calculated according to 2 options:

- option 1: to choose the axial meridian from the average meridian of the network about $90 \mathrm{~km}$, the height of the measured line (height of the reference ellipsoid) $0 \mathrm{~m}$.

- option 2: to apply the proposed coordinate system.

The calculation results are presented in Table 2.

Table 2. Calculation results

\begin{tabular}{|c|c|c|c|c|c|c|c|c|}
\hline \multirow{2}{*}{ No. } & \multicolumn{3}{|c|}{ Line } & \multicolumn{3}{c|}{ UTM System } & \multicolumn{4}{c|}{ The proposed system } \\
\cline { 2 - 9 } & \multicolumn{3}{|c|}{$\begin{array}{c}L_{0}=L_{m}+90 \mathrm{~km} \\
H_{0}=0 \mathrm{~m}\end{array}$} & \multicolumn{3}{c|}{$L_{0}=L_{m} ; H_{0}=637 \mathrm{~m}$} \\
\cline { 2 - 9 } & $\begin{array}{c}\text { Starting } \\
\text { point }\end{array}$ & End point & $Y_{m}, \mathrm{~km}$ & $\Delta S, \mathrm{~mm}$ & $Y_{m}, \mathrm{~km}$ & $\Delta S_{H}$ & $\Delta S_{m p}$ & $\Delta S, \mathrm{~mm}$ \\
\hline $\mathbf{( 1 )}$ & $\mathbf{( 2 )}$ & $\mathbf{( 3 )}$ & $\mathbf{( 4 )}$ & $\mathbf{( 5 )}$ & $\mathbf{( 6 )}$ & $\mathbf{( 7 )}$ & $\mathbf{( 8 )}$ & $(\mathbf{9})$ \\
\hline 1 & 11 & 1 & 580 & -42.4 & 490 & 199.9 & -197.5 & 2.4 \\
\hline 2 & 1 & 2 & 582 & -34.4 & 492 & 199.9 & -198.4 & 1.5 \\
\hline 3 & 2 & 3 & 584 & -26.2 & 494 & 199.9 & -199.1 & 0.8 \\
\hline 4 & 3 & 4 & 586 & -17.9 & 496 & 199.9 & -199.6 & 0.3 \\
\hline 5 & 4 & 5 & 588 & -9.3 & 498 & 199.9 & -199.9 & 0.0 \\
\hline 6 & 5 & 6 & 590 & -0.3 & 500 & 199.9 & -199.9 & 0.0 \\
\hline 7 & 6 & 7 & 592 & 8.4 & 502 & 199.9 & -199.9 & 0.0 \\
\hline 8 & 7 & 8 & 594 & 17.6 & 504 & 199.9 & -199.6 & 0.3 \\
\hline 9 & 8 & 9 & 596 & 27.0 & 506 & 199.9 & -199.1 & 0.8 \\
\hline 10 & 9 & 10 & 598 & 36.5 & 508 & 199.9 & -198.4 & 1.5 \\
\hline 11 & 10 & 12 & 600 & 46.3 & 510 & 199.9 & -197.5 & 2.4 \\
\hline
\end{tabular}

The line length corrections are calculated in table 2 according to option 1 in the column (5) and the results of the line length correction according to option 2 - in the column (9).

Comparison of the data calculated for the two options shows that the line length correction in option 2 (using the proposed coordinate system) has a lower distortion value than in the case of the traditional option. This proves that the geodetic network in the proposed coordinate system has less deformation along the length. 


\section{Conclusion}

The article proposes an improved coordinate system for creating geodetic reference networks during construction using the UTM projection and provides its justification. The formula for calculating the line length correction given in the article is based on theoretical analysis, verified by the experimental calculations and is easily applied in practice. Using the proposed coordinate system it is possible to create geodetic control networks for construction with the smallest length deformation.

\section{References}

1. Chan Thanh Shon, A.A. Kuzin, Analysis of the Earth curvature influence on the results of satellite and traditional measurements in the topocentric coordinate system $\mathbf{6}, 38$ 43 (2018).

2. V.N. Balandin, M.Ya. Bryn, I.V. Menshikov, Yu.G. Firsov, S.L. Shtren, Solution of geodesy and cartography problems in the functions of spatial rectangular coordinates, (Information and Publishing Center of the Government of St. Petersburg "Petrocenter", St. Petersburg, 2013).

3. V.N. Balandin, A.I. Efanov, I.V. Menshikov, Yu.G. Firsov, Conversion of geodetic coordinates to flat rectangular for a wide coordinate zone of the Gaussian projection, Geodesy and Cartography 8, 21-23 (2014).

4. BC 126.13330.2012 Geodetic works in construction. Updated edition of SNiP 3.01.0384. Application date: 26.11.2013.

5. E.B. Klyushin, I.M. Kravchuk, Chin Thanh Truong, Chan Quang Hock, Taking into account the influence of the curvature of the Earth in engineering and geodetic works, Universities' News "Geodesy and aerial photography" 1, 11-14 (2012). 